LA-UR- $09-00733$

Approved for public release;

distribution is unlimited.
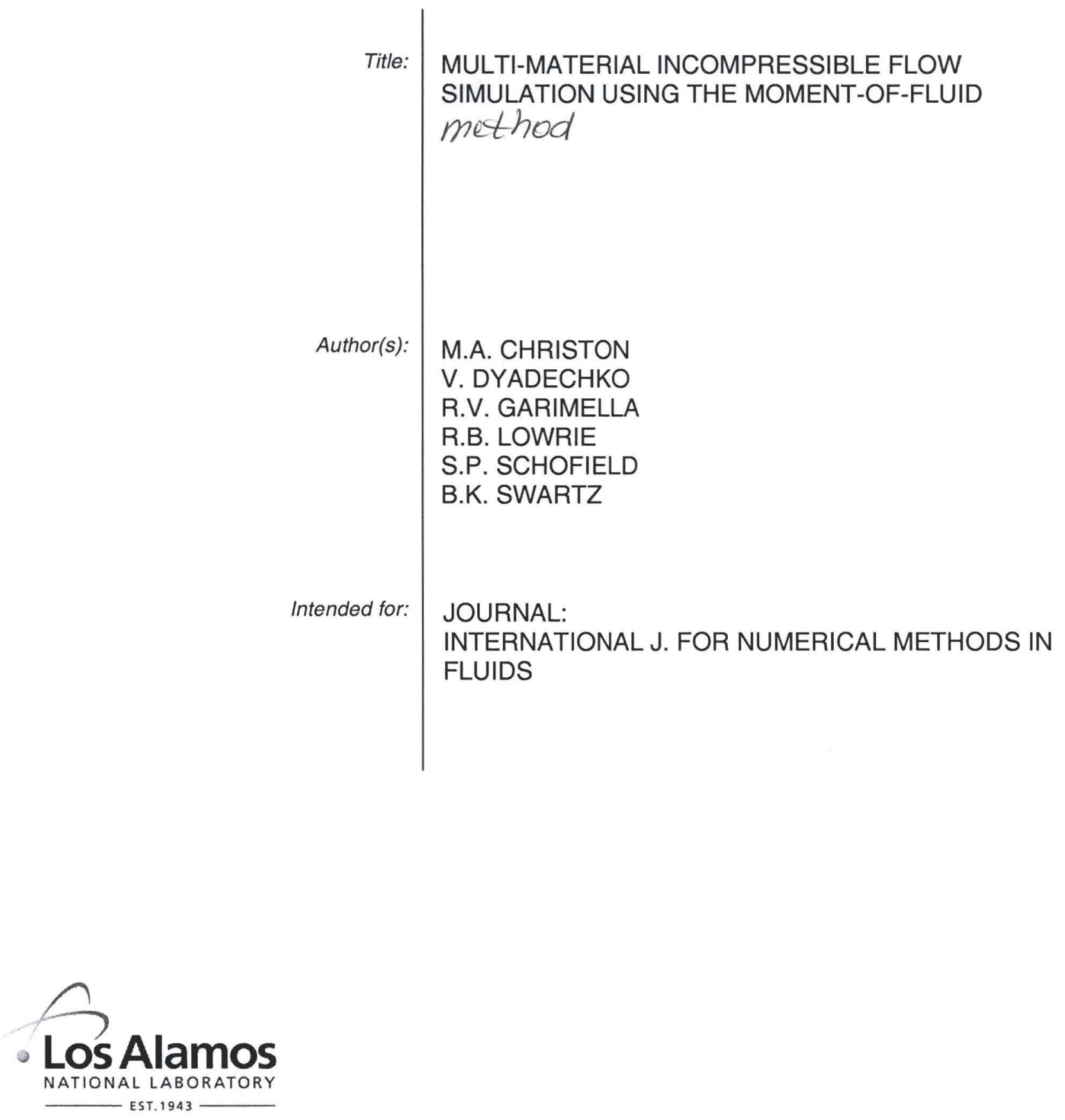

Los Alamos National Laboratory, an affirmative action/equal opportunity employer, is operated by the Los Alamos National Security, LLC for the National Nuclear Security Administration of the U.S. Department of Energy under contract DE-AC52-06NA25396. By acceptance of this article, the publisher recognizes that the U.S. Government retains a nonexclusive, royalty-free license to publish or reproduce the published form of this contribution, or to allow others to do so, for U.S. Government purposes. Los Alamos National Laboratory requests that the publisher identify this article as work performed under the auspices of the U.S. Department of Energy. Los Alamos National Laboratory strongly supports academic freedom and a researcher's right to publish; as an institution, however, the Laboratory does not endorse the viewpoint of a publication or guarantee its technical correctness.

Form $836(7 / 06)$ 


\title{
Multi-material incompressible flow simulation using the Moment-of-Fluid method
}

\author{
M.A. Christon ${ }^{1}$, V. Dyadechko ${ }^{2}$, R. V. Garimella ${ }^{3}$, \\ R. B. Lowrie ${ }^{4}$, S. P. Schofield ${ }^{3}$, B. K. Swartz ${ }^{3}$ \\ 1. SIMULIA
}

2. Exxon-Mobil

3. Applied Mathematics and Plasma Physics (T-5), LANL

4. Computational Physics and Methods (CCS-2), LANL

January 28, 2009

\section{Abstract}

The Moment-of-Fluid interface reconstruction technique is implemented in a second order accurate, unstructured finite element variable density incompressible Navier-Stokes solver. For flows with multiple materials, MOF significantly outperforms existing first and second order interface reconstruction techniques. For two material flows, the performance of MOF is similar to other interface reconstruction techniques. For strongly driven bouyant flows, the errors in the flow solution dominate and all the interface reconstruction techniques perform similarly.

\section{Introduction}

Incompressible flow is one of the most frequently encountered flow regimes encompassing problems that range from atmospheric dispersal to food processing, aerodynamic design of automobiles, and manufacturing processes such as chemical vapor deposition, mold filling and casting. There is an increasing need for multi-material incompressible flow simulations where multiple fluids interact. These cases arise in immiscible fluids and transport problems where the diffusion time scale is far slower than the advection and viscous time scales.

In this work, we demonstrate a second order accurate finite element incompressible viscous flow solver using the moment-of-fluid (MOF) method to implicitly define material and interface positions. The interfaces show true second order convergence and higher accuracy than 
existing first or second order interface reconstruction methods.

A number of interface methods have developed for flow simulations. In interface tracking $\rightarrow$ methods, the position of the interface is explicitly tracked in the flow simulation [4]. In level set methods, the interface position is implicitly defined as the 0 level curve of a distance function [17]. In volume-of-fluids (VOF) methods [20], the fractional volume of each material in a cell is tracked and, when needed, the interface is reconstructed from the volume fraction data. VOF methods are explicitly conservative and can simply manage dramatic topological changes in the interface structure. As a result, VOF methods are extensively used in research and production codes and are the subject of this article.

Fundamental to VOF methods is the interface reconstruction process. Most contemporary reconstruction methods are piecewise linear interface reconstruction (PLIC) methods where the interface in each cell is defined by a line or arbitrary orientation, although alternative methods such as power diagram based reconstruction $[22,23]$ or piecewise parabolic methods (CITE LLNL) have been developed. The original PLIC method developed by Youngs [25, 26] defined the outward interface normal as the negative gradient of the volume fraction function. It is first order accurate, but gives reliable results. Second order accurate methods have been developed including the LVIRA method [14] and Swartz smoothing [24]. Recently, a new second order accurate method, the Moment-of-Fluid (MOF) method [7, 8], has been developed. Unlike LVIRA or Swartz smoothing which utilize only the volume fraction data, MOF achieves second order accuracy by tracking and using the first moments of each fluid region in a cell.

In this article, the MOF method is used in volume tracked incompressible, viscous flow simulations and compared with existing interface reconstructions techniques including Youngs' method, LVIRA, Swartz Smoothing, and the first order power diagram.

\section{Mathematical formulation}

The governing equations are the variable-density incompressible Navier-Stokes equations.

$$
\begin{aligned}
\rho\left(\frac{\partial \mathbf{u}}{\partial t}+\mathbf{u} \cdot \nabla \mathbf{u}\right) & =-\nabla p+\nabla \cdot(\mu \nabla \mathbf{u})+\rho g+F \\
\nabla \cdot \mathbf{u} & =0 \\
\frac{\partial \rho}{\partial t}+\mathbf{u} \cdot \nabla \rho & =0
\end{aligned}
$$

A theoretical treatment of the equations is presented by Danchin [6]. Numerical methods for the solution of the equations with continuous density have been presented by a number of authors $[1,2,3,10,12,16,19]$. Additional methods and results have been presented for simulations utilizing level sets [15] and volume tracking [21, 18]. 
In the case of volume-of-fluids methods, the fractional volume of each fluid in a computational cell is tracked rather than tracking the density (or viscosity) directly. In which, the evolution of the volume fraction function becomes,

$$
\frac{\partial \mathcal{F}_{k}}{\partial t}+\mathbf{u} \cdot \nabla \mathcal{F}_{k}=0 \quad k=1, \ldots, N_{m}
$$

with the density given as

$$
\rho=\sum_{k=1}^{N_{m}} \mathcal{F}_{k} \rho_{k}
$$

where $\mathcal{F}_{k}$ and $\rho_{k}$ are respectively the volume fraction and density of material $k$.

\section{Time discretization}

The time discretization uses a fractional step method

$$
\begin{aligned}
\frac{1}{\Delta t}\left(\mathbf{u}^{n+1}-\mathbf{u}^{n}\right) & =-\mathbf{u}^{n} \cdot \nabla \mathbf{u}^{n}-\frac{1}{2} \frac{1}{\rho^{n+\frac{1}{2}}}\left(\nabla p^{n+1}+\nabla p^{n}\right) \\
& +\frac{1}{2} \frac{1}{\rho^{n+\frac{1}{2}}} \nabla \cdot\left(\mu^{n+\frac{1}{2}}\left(\nabla \mathbf{u}^{n+1}+\nabla \mathbf{u}^{n}\right)\right)+\mathbf{g}
\end{aligned}
$$

The first step computes the momentum update, resulting in a non-divergence free intermediate velocity $\mathbf{u}^{*}$ :

$$
\begin{aligned}
\frac{1}{\Delta t}\left(\mathbf{u}^{*}-\mathbf{u}^{n}\right) & =-\mathbf{u}^{n} \cdot \nabla \mathbf{u}^{n}-\frac{1}{2} \frac{1}{\rho^{n+\frac{1}{2}}} \nabla p^{n} \\
& +\frac{1}{2} \frac{1}{\rho^{n+\frac{1}{2}}} \nabla \cdot\left(\mu^{n+\frac{1}{2}}\left(\nabla \mathbf{u}^{*}+\nabla \mathbf{u}^{n}\right)\right)+\mathbf{g}
\end{aligned}
$$

In Equation $7, \rho^{n+\frac{1}{2}}$ and $\mu^{n+12}$ are derived from the volume fraction data at time $t^{n+\frac{1}{2}}$ which is not available. As such, a second order predictor step using time extrapolation is used to obtain an estimate of $\mathbf{u}^{n+1}$. Using that estimate, the volume fractions are updated with the material update scheme detailed in section 4.1. The volume fractions are then averaged to obtain

$$
\mathcal{F}_{k}^{n+\frac{1}{2}}=\frac{1}{2}\left(\mathcal{F}_{k}^{n}+\mathcal{F}_{k}^{n+1}\right)
$$

Once the initial momentum update is calculates with equation 7 , the second (projection) step in the fractional step method is done:

$$
\begin{aligned}
\frac{1}{\Delta t}\left(\mathbf{u}^{n+1}-\mathbf{u}^{*}\right) & =-\frac{1}{\rho^{n+1}} \nabla p^{n+1} \\
\nabla \cdot \mathbf{u}^{n+1} & =0
\end{aligned}
$$


Taking the divergence of equation 9 we obtain the variable density pressure Poisson equation

$$
\nabla \cdot \mathbf{u}^{*}=\Delta t \nabla \cdot\left(\frac{1}{\rho^{n+\frac{1}{2}}} \nabla p^{n+1}\right)
$$

After solving equation 11, the velocity is updated as

$$
\mathbf{u}^{n+1}=\mathbf{u}^{*}-\Delta t \frac{1}{\rho^{n+\frac{1}{2}}} \nabla p^{n+1}
$$

\section{Spatial discretization}

The weak formulation of Equation 7 is

$$
\begin{aligned}
\int_{\Omega} \rho^{n+\frac{1}{2}}\left(\mathbf{u}^{*}-\mathbf{u}^{n}\right) \varphi d \Omega & =-\Delta t \int_{\Omega} \rho^{n+\frac{1}{2}} \mathbf{u}^{n} \cdot \nabla \mathbf{u}^{n} \varphi d \Omega-\int_{\Omega}\left(\nabla p^{n}\right) \varphi d \Omega \\
& -\int_{\Omega} \mu^{n+\frac{1}{2}} \nabla \varphi \cdot\left(\nabla \mathbf{u}^{*}+\nabla \mathbf{u}^{n}\right) d \Omega+\int_{\Omega} \rho^{n+\frac{1}{2}} \mathbf{g} \varphi d \Omega
\end{aligned}
$$

The spatial discretization uses Q1P0 (or Q1Q0) finite elements. The velocity is expanded in terms of the bilinear nodal basis functions $\varphi_{i}$. The pressure is expressed by piecewise constant basis functions $\bar{\varphi}_{m}$. We define the time dependent element mass matrix

$$
M_{e, i j}^{n+\frac{1}{2}}=\int_{\Omega} \bar{\rho}^{n+\frac{1}{2}} \varphi_{i} \varphi_{j} d \Omega
$$

where $\bar{\rho}^{n+\frac{1}{2}}=\sum_{k=1}^{N_{m}} \mathcal{F}_{k}^{n+\frac{1}{2}} \rho_{k}$. Similarly define the time dependent stiffness matrix

$$
K_{e, i j}^{n+\frac{1}{2}}=\int_{\Omega} \bar{\mu}^{n+\frac{1}{2}} \nabla \varphi_{i} \cdot \nabla \varphi_{j} d \Omega
$$

Following [9], the element average viscosity is computed as

$$
\frac{1}{\bar{\mu}^{n+\frac{1}{2}}}=\sum_{k=1}^{N_{m}} \frac{\mathcal{F}_{k}^{n+\frac{1}{2}}}{\mu_{k}}
$$

We define the operator

$$
\mathcal{A}(\rho ; \mathbf{u}, \varphi) \mathbf{u}=\int_{\Omega}\left(\rho^{n+\frac{1}{2}} \mathbf{u} \cdot \nabla \mathbf{u}\right) \varphi_{i} d \Omega
$$

With the assembled operators, Equation 13 becomes

$$
\begin{aligned}
\left(M^{n+\frac{1}{2}}+\Delta t K^{n+\frac{1}{2}}\right) \mathbf{u}^{*} & =\left(M^{n+\frac{1}{2}}-\Delta t K^{n+\frac{1}{2}}\right) \mathbf{u}^{n} \\
& -\Delta t A\left(\rho^{n+\frac{1}{2}} ; \mathbf{u}^{n}\right) \mathbf{u}^{n} \\
& -\left(M_{L}^{n+\frac{1}{2}}\right)^{-1} M^{n+\frac{1}{2}} B p^{n}+M^{n+\frac{1}{2}} \mathbf{g}
\end{aligned}
$$


where, for consistency, the $\left(M_{L}^{n+\frac{1}{2}}\right)^{-1} M^{n+\frac{1}{2}}$ is applied to the pressure gradient [11].

The pressure Poisson equation (Equation 11) becomes

$$
B^{T} \mathbf{u}^{*}=\Delta t B^{T}\left(M_{L}^{n+\frac{1}{2}}\right)^{-1} B p^{n+1}
$$

And the velocity update is

$$
\mathbf{u}^{n+1}=\mathbf{u}^{*}-\Delta t\left(M_{L}^{n+\frac{1}{2}}\right)^{-1} B p^{n+1}
$$

To eliminate the well known checker board pressure mode from the Q1P0 finite elements, the pressure stabilization of Hughes and Franca is used [5, 13].

\subsection{Material update}

The material update is the semi-Lagrangian scheme detailed by Dyadechko and Shashkov [8].

\section{$5 \quad$ Perturbation sensitivity}

Of particular concern is the sensitivity of MOF and the material and centroid update scheme to the presence of numerical errors in the flow field. For this test we translate a four material disk of radius 0.15 across a $[0,1] \times[0,1]$ domain from initial position of $(0.25,0.25)$ with a velocity of $\mathbf{u}=(1,1)$ to a final time of $t=0.5$. However, a perturbation to the velocity field is added. The first perturbation is solenoidal and is prescribed by the stream function

$$
\psi=\frac{A_{0}}{4 \pi} \sin ^{2}(2 \pi x) \sin ^{2}(2 \pi y)
$$

with the perturbation velocity field then given as $\mathbf{u}^{\prime}=\left(-\frac{\partial \psi}{\partial y}, \frac{\partial \psi}{\partial x}\right)$.

The second perturbation introduces a non-zero divergence in the flow field and is prescribed by

$$
\mathbf{u}^{\prime}=A_{0} \sin (2 \pi x)(1,1)^{T}
$$

The third perturbation is a random perturbation of each nodal velocity uniformly distributed over the range $\left(-A_{0}, A_{0}\right)$.

With all of these perturbations, the final configuration of the four material circle should identical to the initial condition but translated to location $(0.75,0.75)$. As is evident in Figure 1, a perturbation of 20 percent of the based velocity does not significantly change the results. In particular, MOF shows no more sensitivity to the perturbations than the other methods. 


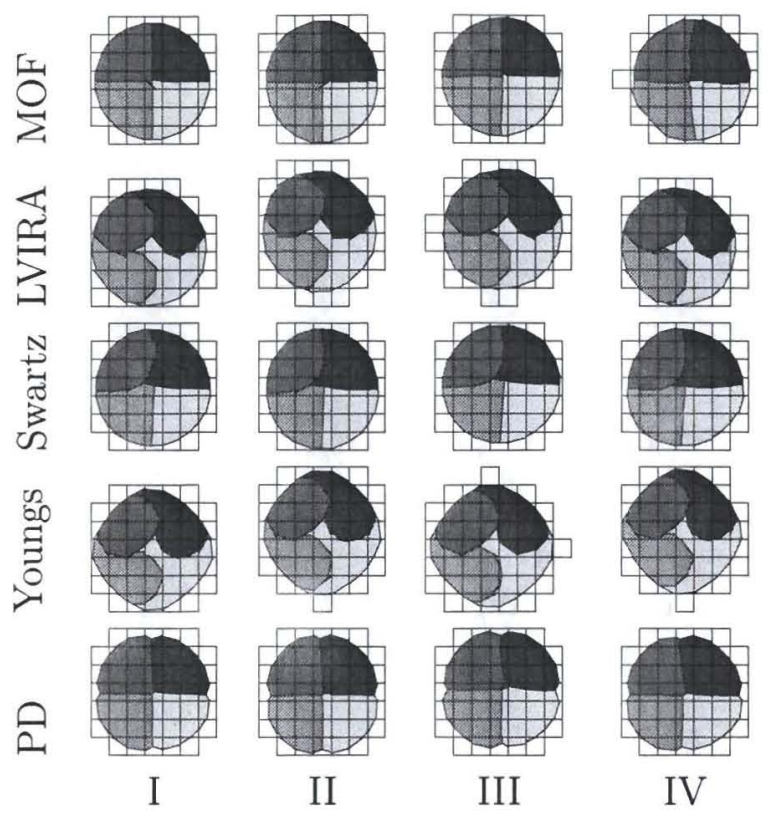

Figure 1: The final configuration of a four material circle after translation with a velocity of $\mathbf{u}=(1,1)^{T}+0.20 \mathbf{u}^{\prime}$ to a final time of $t=0.5$. The perturbations, $\mathbf{u}^{\prime}$, are I. Unperturbed, II. Solenoidal III. Sine with $\nabla \cdot \mathbf{u}^{\prime} \neq 0$ and IV. Random.

\section{$6 \quad$ Incompressible, scalar transport tests}

\subsection{Time reversal}

In the first test case given in [23]. the velocity profile is given by the stream function in a $[0,1] \times[0,1]$ domain

$$
\psi=\frac{1}{\pi} \cos \left(\frac{\pi}{4} t\right) \sin ^{2}(\pi x) \sin ^{2}(\pi y)
$$

The initial condition is a four material circle of radius 0.15 centered at $(0.5,0.75)$. The simulation is run to a final time of $t=4$. At the final time, it should exactly match the initial condition. As shown in Figures 2 and 3, MOF clearly demonstrates superior accuracy on the $64 \times 64$ grid. Indeed, the errors as measured by the area of the symmetric difference provided in table 1 are consistently less than 50 percent of the errors of the second order LVIRA method and first order Youngs method as shown in Table 1. In addition, MOF has none of the material order dependency of LVIRA and Youngs and is much more accurate than the first order, material order independent power diagram method.

For these simulations, the velocity field was imposed at each time step at the nodes and interpolated to element interiors with the bilinear basis functions when required for the material update. This is shown in table 2 which shows the velocity "converges" and second order due solely to the interpolation error of the basis functions. 


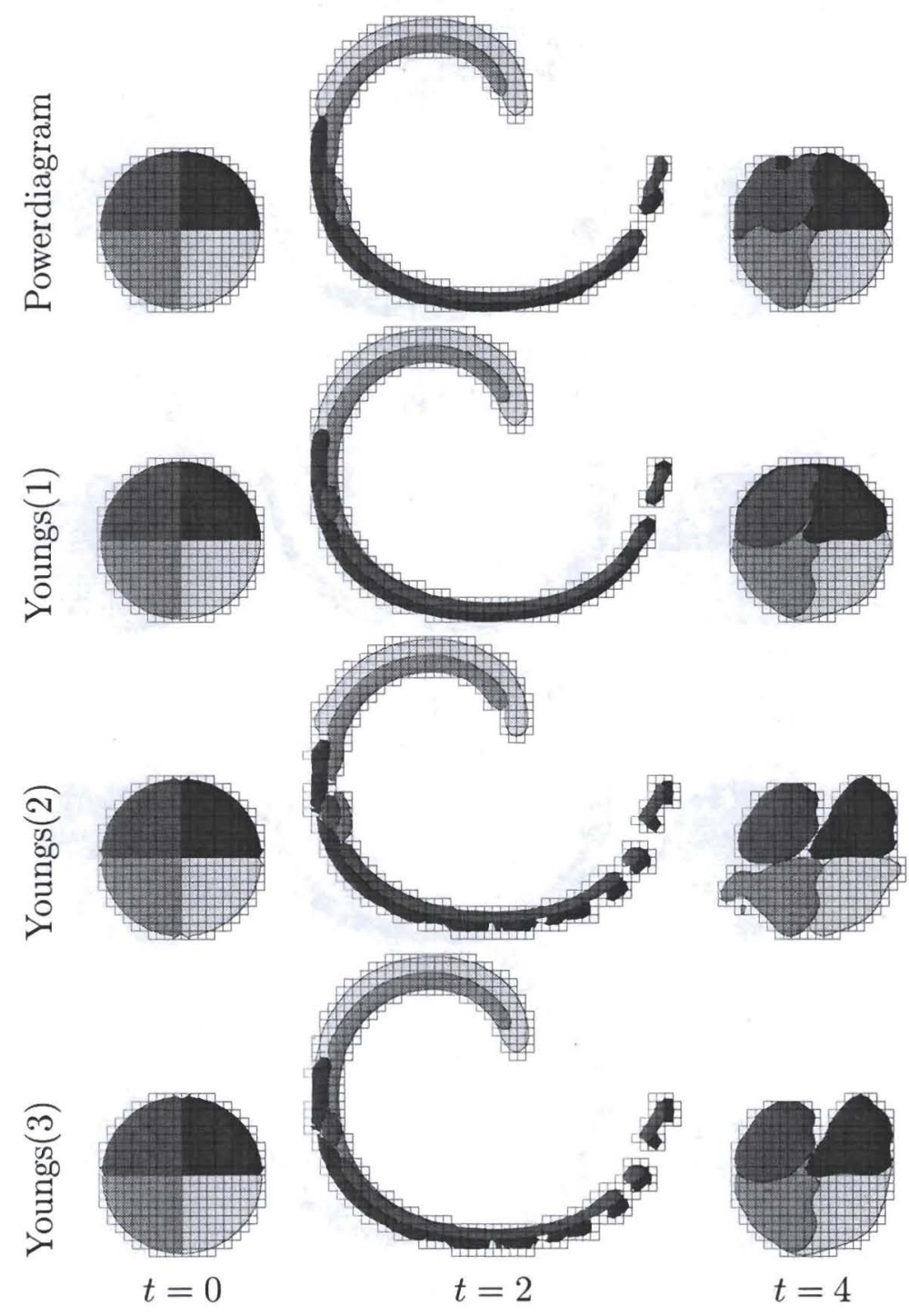

Figure 2: Time reversed vortex. First order methods. 

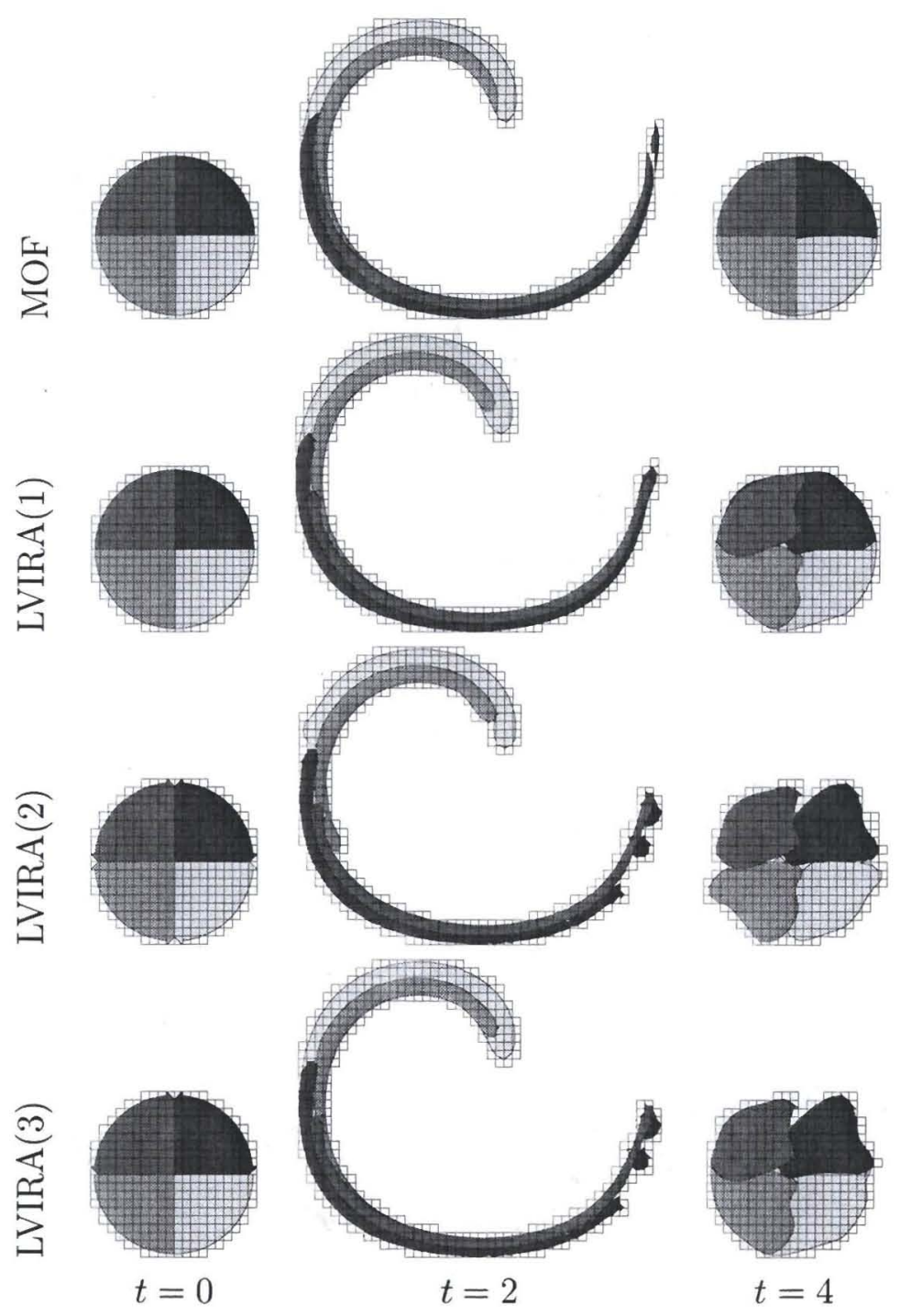

Figure 3: Time reversed vortex. Second order methods 


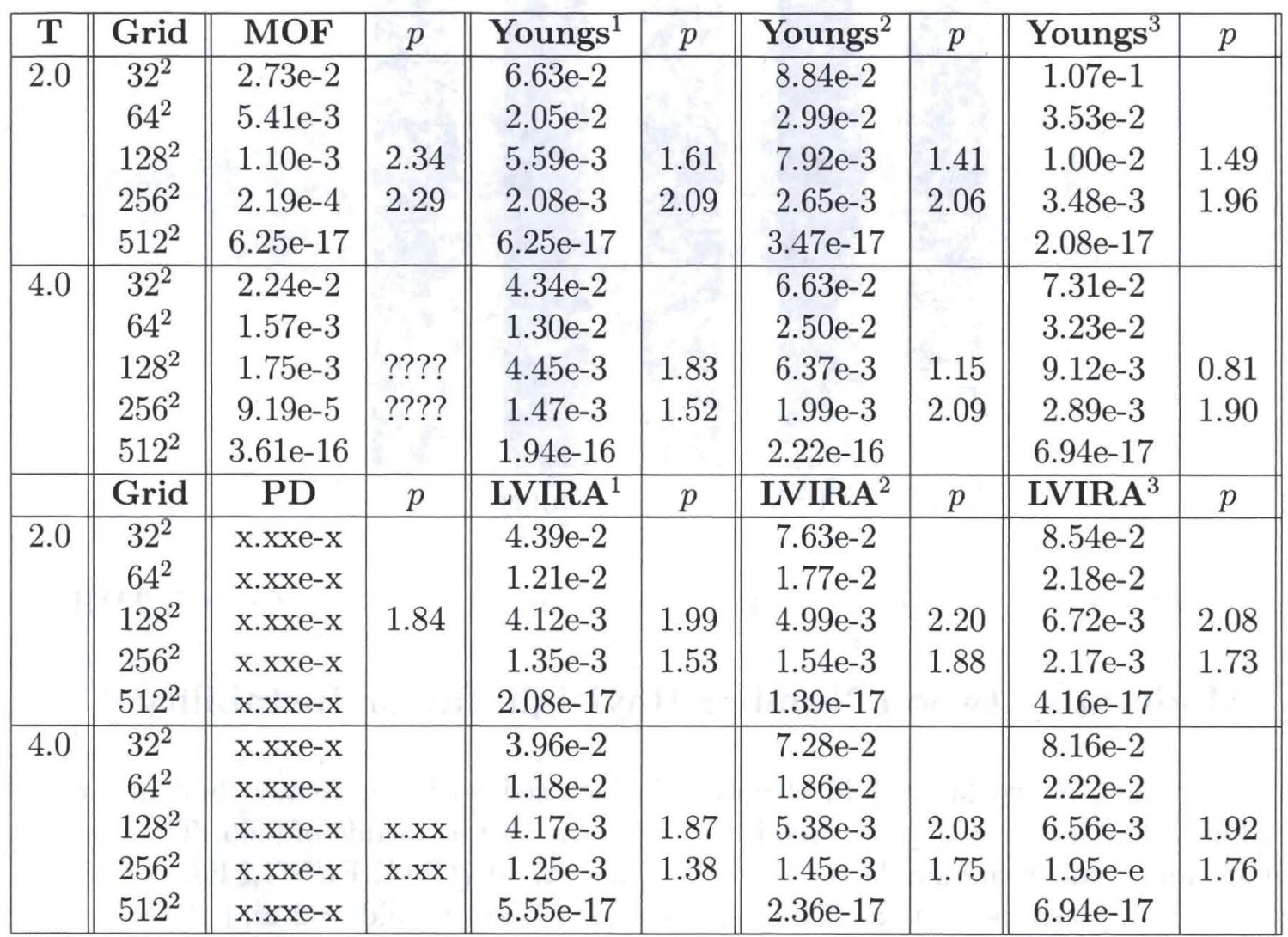

Table 1: CHECK CFL. The area of the symmetric difference error for the vortex-in-a-box test case with a CFL number of $\frac{1}{4}$. (1)-01234, (2)-12034 (3)-12340

\begin{tabular}{|c||c||c|c||c|c||c|c||}
\hline Time & Grid & $\frac{\left\|\mathbf{u}-\mathbf{u}_{512^{2}}\right\|}{\left\|\mathbf{u}_{5122}\right\|}$ & $p$ & $\frac{\left\|\mathbf{u}-\mathbf{u}_{512^{2}}\right\|_{2}}{\left\|\mathbf{u}_{512^{2}}\right\|}$ & $p$ & $\frac{\left\|\mathbf{u}-\mathbf{u}_{512^{2}}\right\|_{\infty}}{\left\|\mathbf{u}_{5122}\right\|}$ & $p$ \\
\hline 2.0 & $32^{2}$ & $4.67 \mathrm{e}-3$ & & $4.81 \mathrm{e}-3$ & & $7.13 \mathrm{e}-3$ & \\
& $64^{2}$ & $1.15 \mathrm{e}-3$ & & $1.19 \mathrm{e}-3$ & & $1.78 \mathrm{e}-3$ & \\
& $128^{2}$ & $2.75 \mathrm{e}-4$ & 2.00 & $2.86 \mathrm{e}-4$ & 2.00 & $4.28 \mathrm{e}-4$ & 1.99 \\
& $256^{2}$ & $5.52 \mathrm{e}-5$ & 2.00 & $5.89 \mathrm{e}-5$ & 2.00 & $8.91 \mathrm{e}-5$ & 2.00 \\
& $512^{2}$ & $4.26 \mathrm{e}-16$ & & $4.80 \mathrm{e}-16$ & & $1.28 \mathrm{e}-15$ & \\
\hline \multirow{2}{*}{4.0} & $32^{2}$ & $4.67 \mathrm{e}-3$ & & $4.81 \mathrm{e}-3$ & & $7.13 \mathrm{e}-3$ & \\
& $64^{2}$ & $1.15 \mathrm{e}-3$ & & $1.19 \mathrm{e}-3$ & & $1.78 \mathrm{e}-3$ & \\
& $128^{2}$ & $2.75 \mathrm{e}-5$ & 2.00 & $2.86 \mathrm{e}-4$ & 2.00 & $4.28 \mathrm{e}-4$ & 1.99 \\
& $256^{2}$ & $5.52 \mathrm{e}-5$ & 2.00 & $5.89 \mathrm{e}-5$ & 2.00 & $8.91 \mathrm{e}-5$ & 2.00 \\
& $512^{2}$ & $4.27 \mathrm{e}-16$ & & $4.79 \mathrm{e}-16$ & & $1.28 \mathrm{e}-15$ & \\
\hline
\end{tabular}

Table 2: The velocity "convergence". Here the velocity is imposed at the nodes from a prescribed analytic formula each time step. As a result, the velocity errors should be associated entirely with the interpolation error of the Q1 basis functions. velocity. 


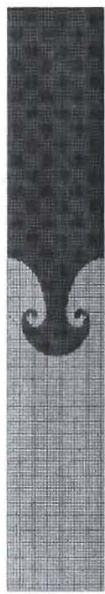

MOF

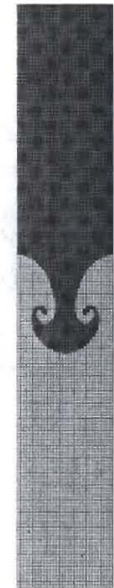

LVIRA

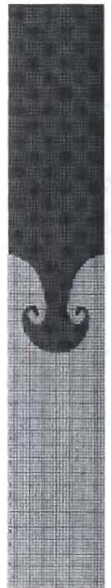

Swartz

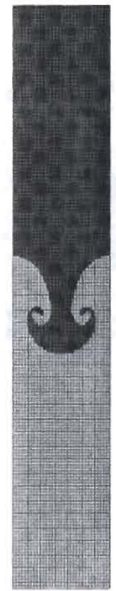

Youngs

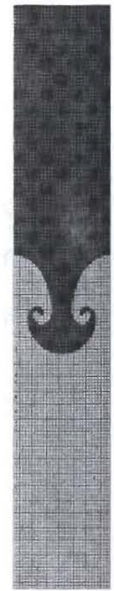

$\mathrm{PD}$

Figure 4: Moderate Atwood Number Rayleigh-Taylor at time $t=0.1$ CHECK TIME

\subsection{Moderate Atwood Number Rayleigh-Taylor Instability}

In this test case, a two layer fluid structure is imposed with the heavy fluid above the lighter fluid. The interface is perturbed by a cosine with a magnitude of 0.05 . The material properties and domain are similar to those by Fung et. at (CITE FUNG,FRANCOIS,etc) but in an incompressible context. The density of the heavier fluid is 2 and the molecular viscosity $\mu=0.01$, the lighter fluid has a density of 1 and viscosity $\mu=1.0 e-4$. The domain is $[0,1] \times[-3,3]$. The simulation was run on $32 \times 192,64 \times 384$, and $128 \times 768$ using fixed time steps of $1.0 e-4,0.5 e-4$, and $0.25 e-4$ respectively. The results are shown in figure 4 .

\subsection{Air-Helium Rayleigh-Taylor Instability}

This simulation is similar to the Rayleigh-Taylor simulations in section 6.2. However, the material parameters and domain size are different. In Puckett, et. al. [18], the two fluids chosen were air and helium. The air has density $1.225 \mathrm{~kg} / \mathrm{m}^{3}$ and viscosity $1.77625 \times 10^{-5}$ $\mathrm{kg} / \mathrm{m} \mathrm{s}$. The helium has density $0.1694 \mathrm{~kg} / \mathrm{m}^{3}$ and viscosity $1.941 \times 10^{-5} \mathrm{~kg} / \mathrm{m} \mathrm{s}$. The domain is $0.01 \mathrm{~m} \times 0.04 \mathrm{~m}$. The interface located at the midplane is perturbed with a single period of a cosine with an amplitude of $5 \times 10^{4} \mathrm{~m}$. As shown in figure 5 , all of the interface reconstruction methods produced similar results. The convergence of the interfaces as measured by the area of the symmetric difference is provided in table 3 . No significant difference is shown between the methods. In table 4 , the convergence of the velocity field is also large unaffected by the choice of interface reconstruction method.

\section{UNSTRUCTURED RESULTS.}



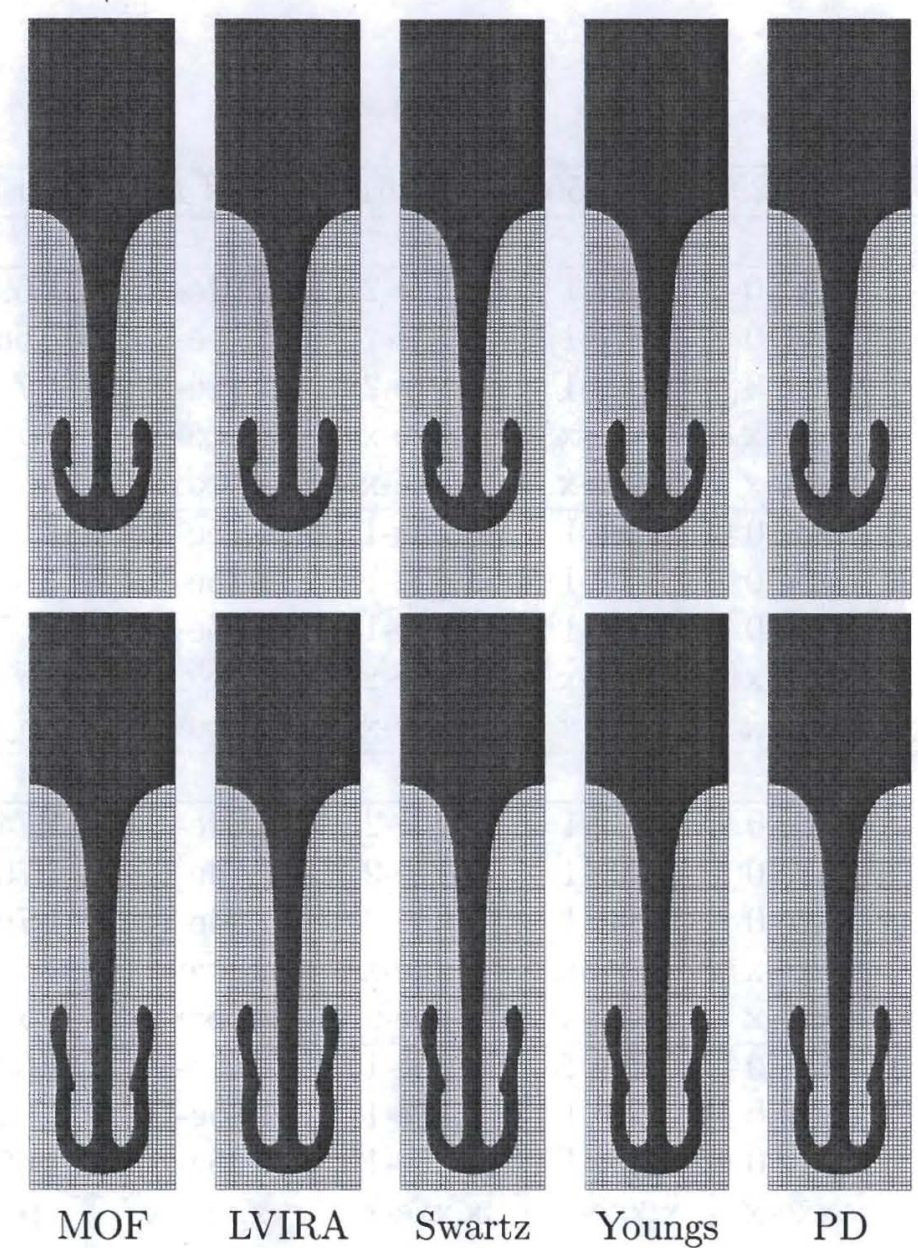

Swartz Youngs

PD

Figure 5: Air-Helium Rayleigh-Taylor at time $t=0.10 \mathrm{~s}$ and $t=0.14 \mathrm{~s}$

\begin{tabular}{|c|c|c|c|c|c|c|c|c|c|c|}
\hline $\mathbf{T}$ & Grid & MOF & $p$ & PD & $p$ & Youngs & $p$ & LVIRA & Swartz & $p$ \\
\hline \multirow[t]{4}{*}{0.10} & $32 \times 128$ & $2.32 \mathrm{e}-2$ & \multirow{4}{*}{1.72} & x.xxe-x & \multirow{4}{*}{$\mathrm{x} \cdot \mathrm{xx}$} & $2.20 \mathrm{e}-2$ & \multirow{4}{*}{1.78} & $2.53 \mathrm{e}-2$ & \multirow{4}{*}{1.66} & x.xxe-x \\
\hline & $64 \times 256$ & $6.61 \mathrm{e}-3$ & & x.xxe-x & & $6.07 \mathrm{e}-3$ & & $7.44 \mathrm{e}-3$ & & x.xxe-x \\
\hline & $128 \times 512$ & $1.58 \mathrm{e}-3$ & & x.xxe-X & & $1.42 \mathrm{e}-3$ & & $1.80 \mathrm{e}-3$ & & x.xxe-X \\
\hline & $256 \times 1024$ & $8.85 \mathrm{e}-17$ & & x.xxe-x & & $4.21 \mathrm{e}-17$ & & $4.81 \mathrm{e}-17$ & & x.xxe-x \\
\hline \multirow[t]{4}{*}{0.14} & $32 \times 128$ & $4.02 \mathrm{e}-2$ & \multirow{4}{*}{1.71} & x.xxe-X & \multirow{4}{*}{$\mathrm{x} . \mathrm{xx}$} & $3.95 \mathrm{e}-2$ & \multirow{4}{*}{1.69} & $4.21 \mathrm{e}-2$ & \multirow{4}{*}{1.73} & x.xxe-x \\
\hline & $64 \times 256$ & $1.16 \mathrm{e}-2$ & & x.xxe-x & & $1.19 \mathrm{e}-2$ & & $1.21 \mathrm{e}-2$ & & x.xxe-x \\
\hline & $128 \times 512$ & $2.83 \mathrm{e}-3$ & & x.xxe-x & & $3.32 \mathrm{e}-3$ & & $3.04 \mathrm{e}-3$ & & x.xxe-X \\
\hline & $256 \times 1024$ & $6.51 \mathrm{e}-19$ & & x.xxe-x & & $8.04 \mathrm{e}-17$ & & $6.33 \mathrm{e}-17$ & & X.xxe-x \\
\hline
\end{tabular}

Table 3: The relative area of the symmetric difference error for the Air-Helium RayleighTaylor problem 


\begin{tabular}{|c|c|c|c|c|c|c|}
\hline Time & Method & $32 \times 128$ & $64 \times 256$ & $128 \times 512$ & $256 \times 1024$ & $p$ \\
\hline \multicolumn{7}{|c|}{$L_{1}$} \\
\hline \multirow[t]{5}{*}{0.10} & MOF & $1.24 \mathrm{e}-0$ & $3.59 \mathrm{e}-1$ & $8.77 \mathrm{e}-2$ & $5.01 \mathrm{e}-14$ & 1.69 \\
\hline & LVIRA & $1.30 \mathrm{e}-0$ & $3.87 \mathrm{e}-1$ & $9.52 \mathrm{e}-2$ & $1.11 \mathrm{e}-14$ & 1.65 \\
\hline & Youngs & $1.20 \mathrm{e}-0$ & $3.37 \mathrm{e}-1$ & $7.91 \mathrm{e}-2$ & $1.11 \mathrm{e}-14$ & 1.74 \\
\hline & Swartz & x.xxe-x & x.xxe-x & X.Xxe-x & x.xxe-x & $\mathrm{p}$ \\
\hline & $\mathrm{PD}$ & x.xxe-x & x.xxe-x & x.xxe-x & x.xxe-x & $\mathrm{p}$ \\
\hline \multirow[t]{5}{*}{0.14} & MOF & $1.83 \mathrm{e}-0$ & $5.48 \mathrm{e}-1$ & $1.35 \mathrm{e}-1$ & $1.75 \mathrm{e}-14$ & 1.63 \\
\hline & LVIRA & $1.89 \mathrm{e}-0$ & $5.75 \mathrm{e}-1$ & $1.42 \mathrm{e}-1$ & $1.75 \mathrm{e}-14$ & 1.61 \\
\hline & Youngs & $1.79 \mathrm{e}-0$ & $5.32 \mathrm{e}-1$ & $1.30 \mathrm{e}-1$ & $1.75 \mathrm{e}-14$ & 1.65 \\
\hline & Swartz & x.xxe-x & x.xxe-x & x.xxe-x & x.xxe-x & $\mathrm{p}$ \\
\hline & $\mathrm{PD}$ & x.xxe-x & x.xxe-x & x.xxe-x & x.xxe-x & $\mathrm{p}$ \\
\hline \multicolumn{7}{|c|}{$L_{2}$} \\
\hline \multirow[t]{5}{*}{0.10} & MOF & $1.25 \mathrm{e}-0$ & $3.54 \mathrm{e}-1$ & $8.45 \mathrm{e}-2$ & $1.04 \mathrm{e}-14$ & 1.74 \\
\hline & LVIRA & $1.31 \mathrm{e}-0$ & $3.80 \mathrm{e}-1$ & $9.17 \mathrm{e}-2$ & $1.04 \mathrm{e}-14$ & 1.70 \\
\hline & Youngs & $1.22 \mathrm{e}-0$ & $3.33 \mathrm{e}-1$ & $7.65 \mathrm{e}-2$ & $1.04 \mathrm{e}-14$ & 1.79 \\
\hline & Swartz & x.xxe-x & x.xxe-x & X.xxe-x & x.xxe-x & $\mathrm{p}$ \\
\hline & $\mathrm{PD}$ & x.xxe-x & x.xxe-x & x.xxe-x & x.xxe-x & $\mathrm{p}$ \\
\hline \multirow[t]{5}{*}{0.14} & MOF & $1.80 \mathrm{e}-0$ & $5.41 \mathrm{e}-1$ & $1.33 \mathrm{e}-1$ & $1.45 \mathrm{e}-14$ & 1.63 \\
\hline & LVIRA & $1.91 \mathrm{e}-0$ & $5.72 \mathrm{e}-1$ & $1.40 \mathrm{e}-1$ & $1.45 \mathrm{e}-14$ & 1.63 \\
\hline & Youngs & $1.81 \mathrm{e}-0$ & $5.32 \mathrm{e}-1$ & $1.27 \mathrm{e}-1$ & $1.45 \mathrm{e}-14$ & 1.65 \\
\hline & Swartz & x.xxe-x & x.xxe-x & X.xxe-X & x.xxe-x & $\mathrm{p}$ \\
\hline & $\mathrm{PD}$ & x.xxe-x & x.xxe-x & x.xxe-x & x.xxe-x & $\mathrm{p}$ \\
\hline \multicolumn{7}{|c|}{$L_{\infty}$} \\
\hline \multirow[t]{5}{*}{0.10} & $\mathrm{MOF}$ & $3.42 \mathrm{e}-0$ & $1.10 \mathrm{e}-0$ & $2.77 \mathrm{e}-1$ & $6.75 \mathrm{e}-14$ & 1.49 \\
\hline & LVIRA & $3.48 \mathrm{e}-0$ & $1.14 \mathrm{e}-0$ & $2.88 \mathrm{e}-1$ & $6.75 \mathrm{e}-14$ & 1.45 \\
\hline & Youngs & $3.36 \mathrm{e}-0$ & $1.07 \mathrm{e}-0$ & $2.68 \mathrm{e}-1$ & $6.75 \mathrm{e}-14$ & 1.51 \\
\hline & Swartz & x.xxe-x & X.xxe-x & x.xxe-X & $\mathrm{x} . \mathrm{xxe}-\mathrm{x}$ & $\mathrm{p}$ \\
\hline & $\mathrm{PD}$ & x.xxe-x & x.xxe-x & X.xxe-X & X.xxe-x & $\mathrm{p}$ \\
\hline \multirow[t]{5}{*}{0.14} & MOF & $5.58 \mathrm{e}-0$ & $1.62 \mathrm{e}-0$ & $3.92 \mathrm{e}-1$ & $7.82 \mathrm{e}-14$ & 1.70 \\
\hline & LVIRA & $5.98 \mathrm{e}-0$ & $1.69 \mathrm{e}-0$ & $4.07 \mathrm{e}-1$ & $7.99 \mathrm{e}-14$ & 1.74 \\
\hline & Youngs & $5.74 \mathrm{e}-0$ & $1.62 \mathrm{e}-0$ & $3.84 \mathrm{e}-1$ & $8.17 \mathrm{e}-14$ & 1.74 \\
\hline & Swartz & x.xxe-x & x.xxe-x & X.xxe-X & X.xxe-x & $\mathrm{p}$ \\
\hline & PD & x.xxe-x & x.xxe-x & x.xxe-x & x.xxe-x & $\mathrm{p}$ \\
\hline
\end{tabular}

Table 4: $L_{1}, L_{2}$, and $L_{\infty}$ error for the $V$ velocity in the Air-Helium Rayleigh-Taylor problem 


\begin{tabular}{|c|c|c|c|c|c|c|c|c|c|c|}
\hline $\mathbf{T}$ & Grid & MOF & $p$ & PD & $p$ & Youngs & $p$ & LVIRA & Swartz & $p$ \\
\hline \multirow[t]{4}{*}{0.05} & $32 \times 64$ & $4.25 \mathrm{e}-2$ & \multirow{4}{*}{1.00} & $4.37 \mathrm{e}-2$ & & $4.35 \mathrm{e}-2$ & \multirow{4}{*}{1.04} & $4.25 \mathrm{e}-2$ & \multirow{4}{*}{1.06} & x.xxe-x \\
\hline & $64 \times 128$ & $1.96 \mathrm{e}-2$ & & $1.98 \mathrm{e}-2$ & & $1.98 \mathrm{e}-2$ & & $1.93 \mathrm{e}-2$ & & x.xxe-x \\
\hline & $128 \times 256$ & $8.13 \mathrm{e}-3$ & & $8.31 \mathrm{e}-3$ & 1.06 & $8.25 \mathrm{e}-3$ & & $8.10 \mathrm{e}-3$ & & x.xxe-x \\
\hline & $256 \times 512$ & $1.39 \mathrm{e}-17$ & & $1.67 \mathrm{e}-16$ & & $1.46 \mathrm{e}-16$ & & $2.08 \mathrm{e}-17$ & & x.xxe-x \\
\hline \multirow[t]{4}{*}{0.06} & $32 \times 64$ & $6.83 \mathrm{e}-2$ & \multirow{4}{*}{1.03} & $6.91 \mathrm{e}-2$ & \multirow{4}{*}{1.09} & $6.80 \mathrm{e}-2$ & & $6.86 \mathrm{e}-2$ & \multirow{4}{*}{0.91} & x.xxe-x \\
\hline & $64 \times 128$ & $3.59 \mathrm{e}-2$ & & $3.63 \mathrm{e}-2$ & & $3.58 \mathrm{e}-2$ & & $3.74 \mathrm{e}-2$ & & x.xxe-x \\
\hline & $128 \times 256$ & $2.00 \mathrm{e}-2$ & & $2.09 \mathrm{e}-2$ & & $1.99 \mathrm{e}-2$ & 1.02 & $3.07 \mathrm{e}-2$ & & x.xxe-x \\
\hline & $256 \times 512$ & $1.04 \mathrm{e}-16$ & & $1.39 \mathrm{e}-17$ & & $8.33 \mathrm{e}-17$ & & 8.33e-17 & & x.xxe-x \\
\hline
\end{tabular}

Table 5: The relative area of the symmetric difference error for the colliding bubbles problem

\subsection{Air bubble Rising in Water}

In this challenging test case also from Puckett, et. al. [18] an air bubble rises in a tank. The domain is $0.7 \mathrm{~cm} \times 1.4 \mathrm{~cm}$. The bubble has a radius of $0.15 \mathrm{~cm}$ and is centered in the domain at a height of $0.25 \mathrm{~cm}$ above the bottom. For the background water, $\rho_{1}=.9992 \mathrm{~g} / \mathrm{cm}^{3}$ and $\mu_{1}=1.1377 \times 10^{-2} \mathrm{~g} / \mathrm{m} \mathrm{s}$. For the air bubble, $\rho_{2}=.001225 \mathrm{~g} / \mathrm{cm}^{3}$ and $\mu_{2}=1.7762 \times 10^{-4}$ $\mathrm{g} / \mathrm{m} \mathrm{s}$. Gravity is taken to be $g=981 \mathrm{~cm} / \mathrm{s}$ along the y axis. There are no surface tension effects.

The results are shown in figures 6 and 7 .

\subsection{Colliding Bubbles}

While MOF does demonstrate improved accuracy for two material simulations, the most significant benefit is shown for simulations containing multiple materials. To demonstrate this, we consider the simple test case of two bubbles water. The physical domain is $[0,1] \times$ $[0,2]$. Two spherical bubbles of radius 0.15 are located at $(0.5,0.25)$ and $(0.5,0.75)$ and have a density of 0.5 and 1.5 respectively. The background fluid has a density of 1.0. All three fluids have a viscosity of 0.01. As shown in Figures 8 and 9 , MOF better preserves the thin material interfaces between the bubbles. However, as illustrated in tables 5 and 6 , the convergence for all of the methods is poor and the errors are essentially identical. This is likely due to the simulation being underresolved so the asymptotic convergence regime has not yet been attained.

\section{Discussion}

For flows with multiple materials, MOF significantly outperforms existing first and second order interface reconstruction techniques. For two material flows, the performance of MOF is similar to other interface reconstruction techniques. As such, for two material flows, MOF 


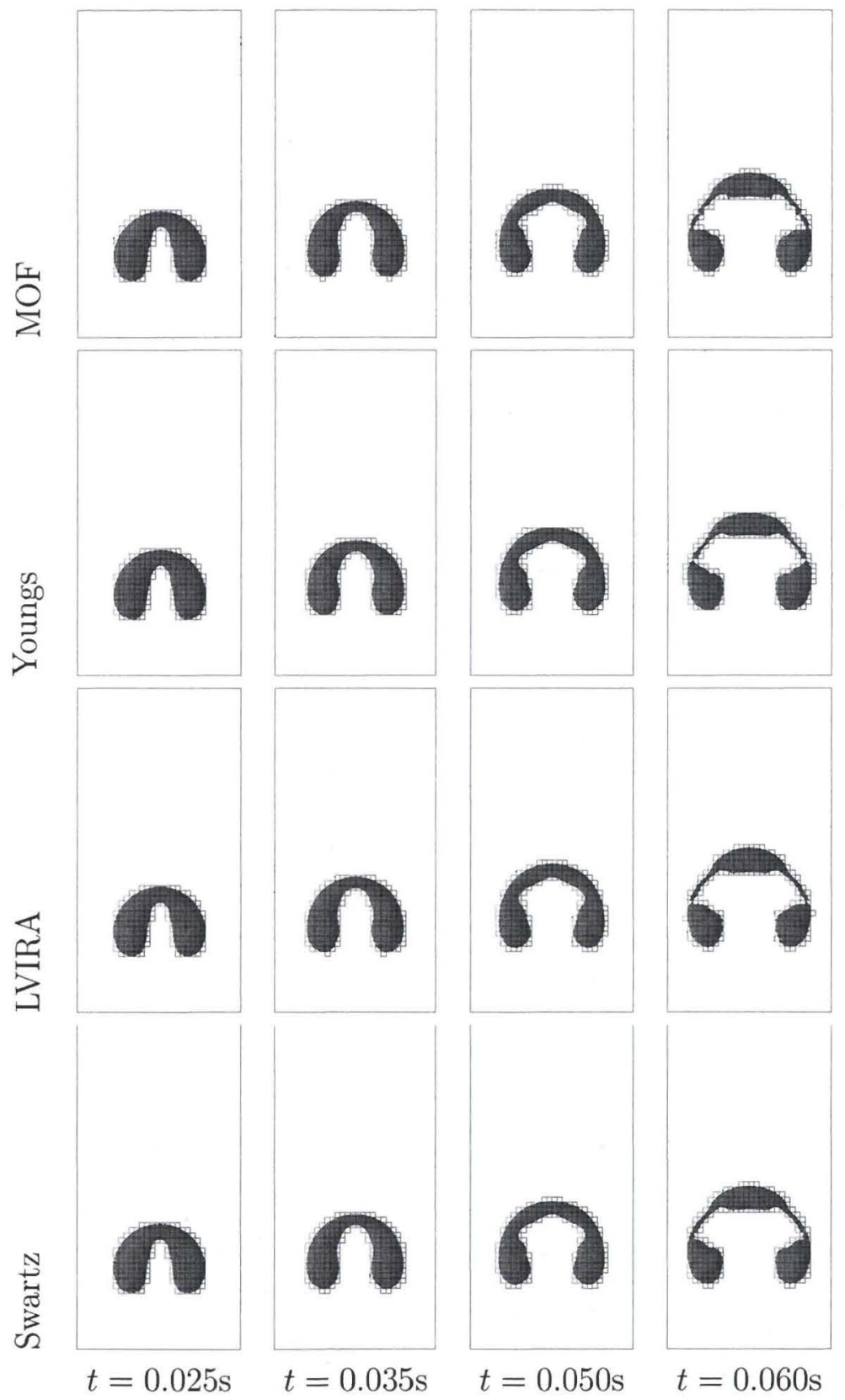

Figure 6: Simulation of an air bubble rising in water with surface tension effects neglected. The grid is $32 \times 64$ 


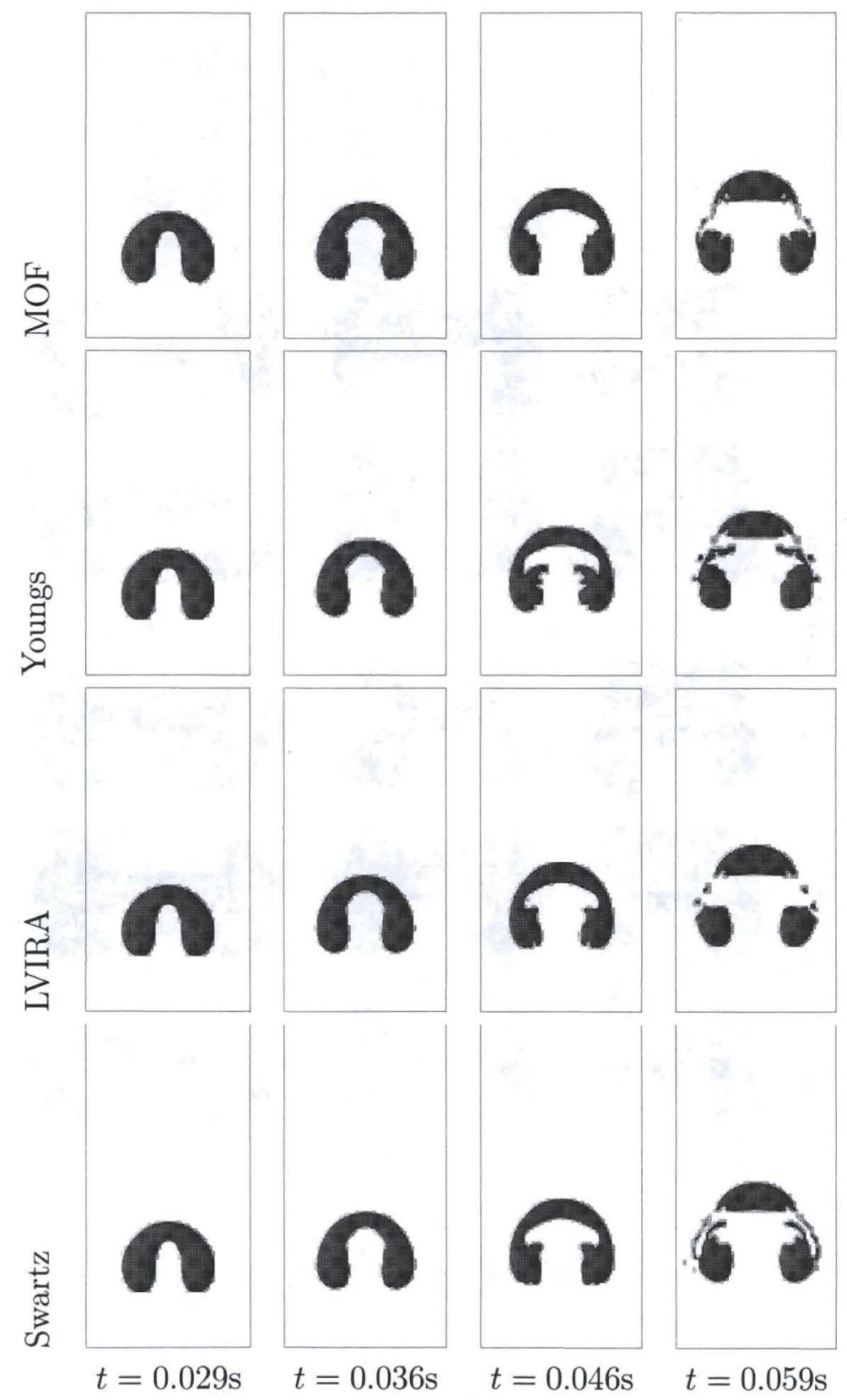

Figure 7: Simulation of an air bubble rising in water with surface tension effects neglected. The grid is $64 \times 128$ 


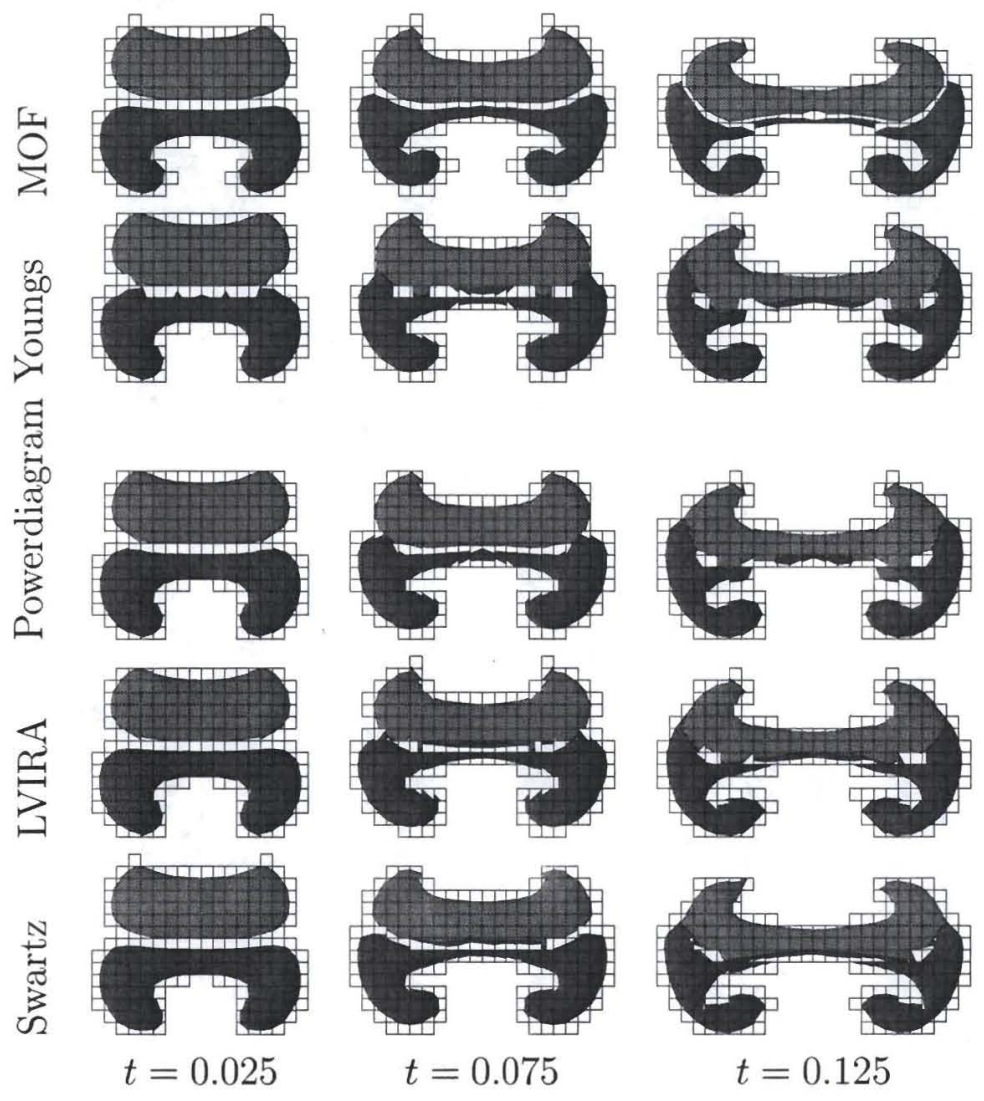

Figure 8: Colliding bubbles on $32 \times 64$ grids. 


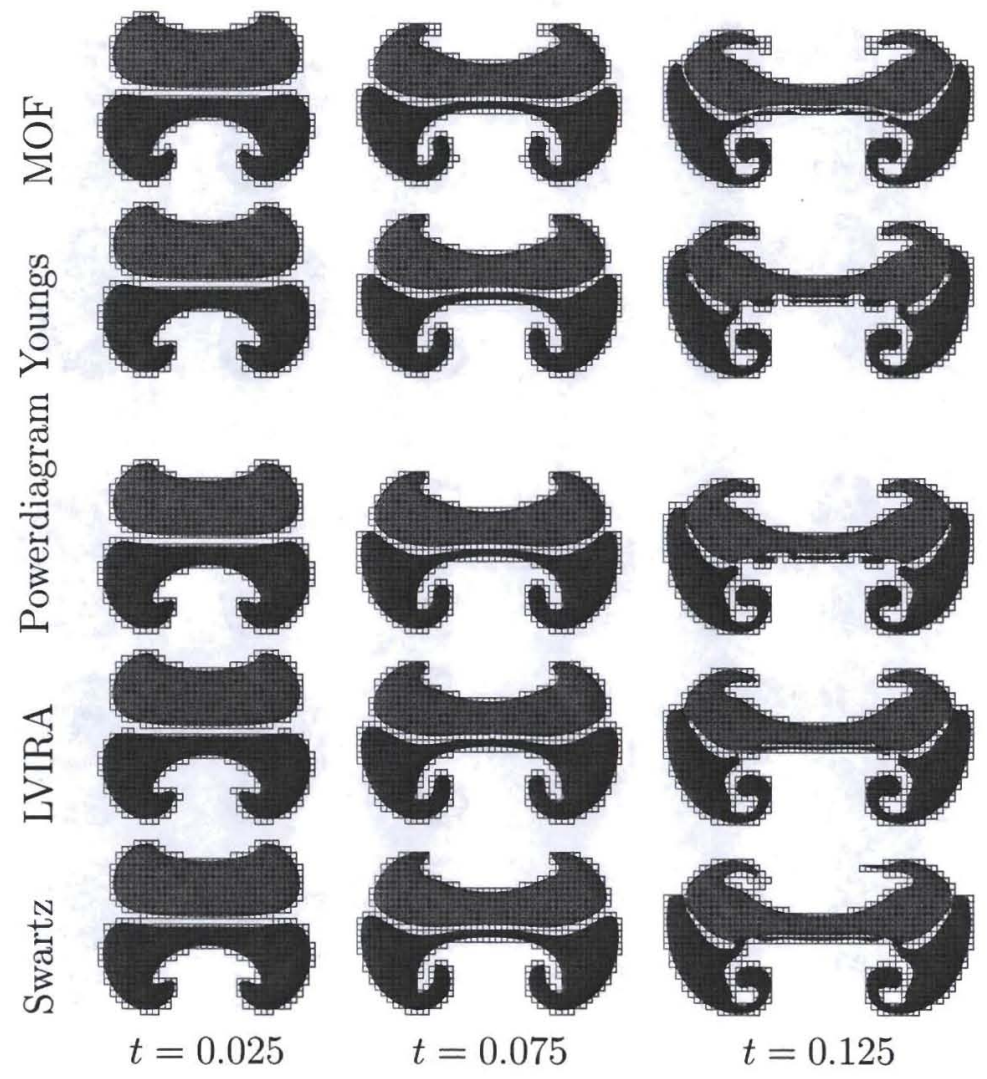

Figure 9: Colliding bubbles on $64 \times 128$ grids. 


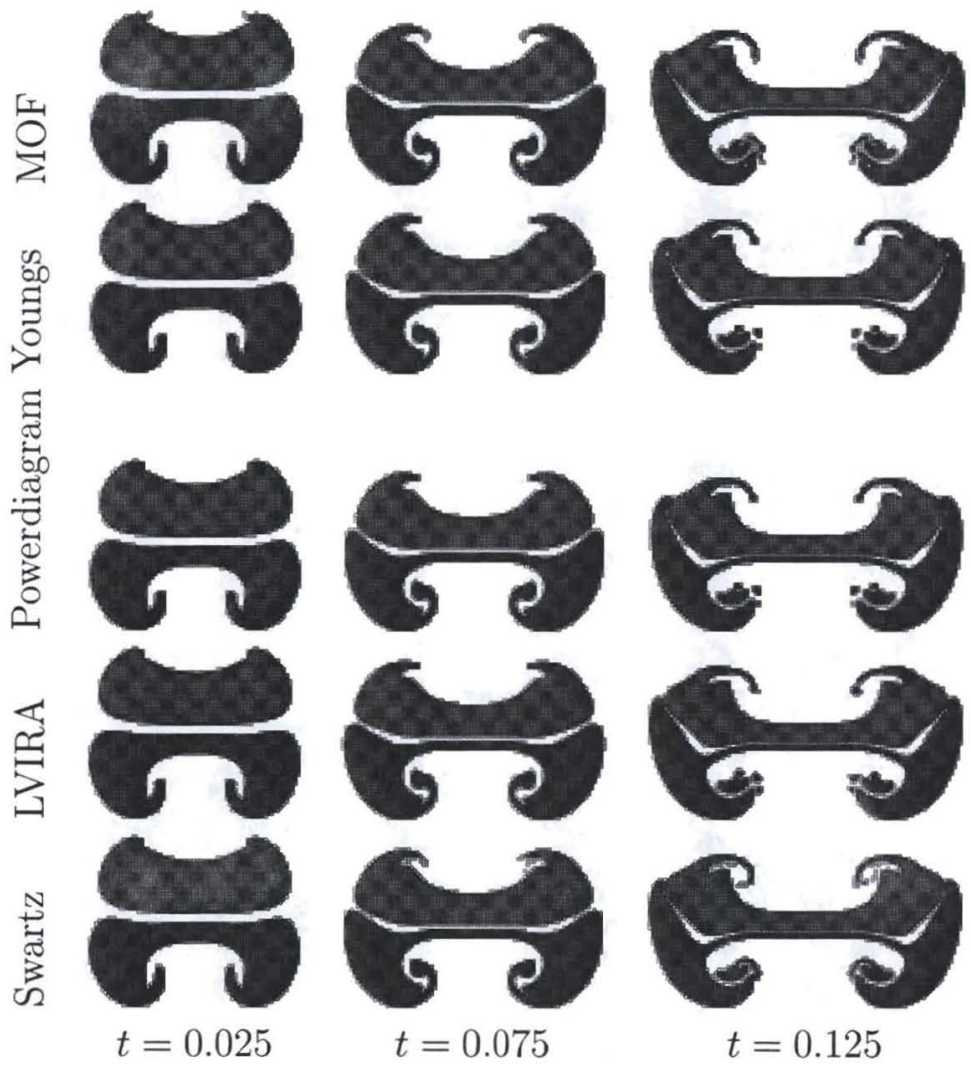

Figure 10: Colliding bubbles on $128 \times 256$ grids. 


\begin{tabular}{|c|c|c|c|c|c|c|}
\hline Time & Method & $32 \times 128$ & $64 \times 256$ & $128 \times 512$ & $256 \times 1024$ & $p$ \\
\hline \multicolumn{7}{|c|}{$L_{1}$} \\
\hline \multirow[t]{5}{*}{0.05} & MOF & $8.96 \mathrm{e}-1$ & $3.78 \mathrm{e}-1$ & $1.14 \mathrm{e}-1$ & $1.13 \mathrm{e}-15$ & 0.97 \\
\hline & LVIRA & $8.96 \mathrm{e}-1$ & $3.78 \mathrm{e}-1$ & $1.14 \mathrm{e}-1$ & $1.13 \mathrm{e}-15$ & 0.97 \\
\hline & Youngs & $8.97 \mathrm{e}-1$ & $3.79 \mathrm{e}-1$ & $1.14 \mathrm{e}-1$ & $1.13 \mathrm{e}-15$ & 0.97 \\
\hline & Swartz & x.xxe-x & x.xxe-x & x.xxe-x & x.xxe-x & $\mathrm{p}$ \\
\hline & PD & $8.97 \mathrm{e}-1$ & $3.79 \mathrm{e}-1$ & $1.14 \mathrm{e}-\mathrm{x}$ & $1.13 \mathrm{e}-15$ & 0.97 \\
\hline \multirow[t]{5}{*}{0.06} & MOF & $1.05 \mathrm{e}-0$ & $4.43 \mathrm{e}-1$ & $1.41 \mathrm{e}-1$ & $1.18 \mathrm{e}-15$ & 1.01 \\
\hline & LVIRA & $1.05 \mathrm{e}-0$ & $4.44 \mathrm{e}-1$ & $1.41 \mathrm{e}-1$ & $1.19 \mathrm{e}-15$ & 1.01 \\
\hline & Youngs & $1.05 \mathrm{e}-0$ & $4.45 \mathrm{e}-1$ & $1.42 \mathrm{e}-1$ & $1.18 \mathrm{e}-15$ & 1.01 \\
\hline & Swartz & x.xxe-x & x.xxe-x & x.xxe-x & x.xxe-x & $\mathrm{p}$ \\
\hline & $\mathrm{PD}$ & $1.05 \mathrm{e}-0$ & $4.46 \mathrm{e}-1$ & $1.43 \mathrm{e}-1$ & $1.19 \mathrm{e}-15$ & 1.00 \\
\hline \multicolumn{7}{|c|}{$L_{2}$} \\
\hline \multirow[t]{5}{*}{0.05} & MOF & $9.17 \mathrm{e}-0$ & $5.01 \mathrm{e}-1$ & $1.73 \mathrm{e}-1$ & $1.93 \mathrm{e}-15$ & 0.34 \\
\hline & LVIRA & $9.16 \mathrm{e}-1$ & $5.00 \mathrm{e}-1$ & $1.73 \mathrm{e}-1$ & $1.93 \mathrm{e}-15$ & 0.34 \\
\hline & Youngs & $9.17 \mathrm{e}-0$ & $5.02 \mathrm{e}-1$ & $1.74 \mathrm{e}-1$ & $1.94 \mathrm{e}-14$ & 0.34 \\
\hline & Swartz & x.xxe-x & x.xxe-x & x.xxe-x & x.xxe-x & $\mathrm{p}$ \\
\hline & PD & $9.18 \mathrm{e}-0$ & $5.02 \mathrm{e}-1$ & $1.74 \mathrm{e}-1$ & $1.94 \mathrm{e}-15$ & 0.34 \\
\hline \multirow[t]{5}{*}{0.06} & MOF & $1.07 \mathrm{e}-0$ & $5.77 \mathrm{e}-1$ & $2.08 \mathrm{e}-1$ & $2.06 \mathrm{e}-15$ & 0.42 \\
\hline & LVIRA & $1.07 \mathrm{e}-0$ & $5.77 \mathrm{e}-1$ & $2.07 \mathrm{e}-1$ & $2.06 \mathrm{e}-15$ & 0.42 \\
\hline & Youngs & $1.07 \mathrm{e}-0$ & $5.78 \mathrm{e}-1$ & $2.09 \mathrm{e}-1$ & $2.07 \mathrm{e}-15$ & 0.42 \\
\hline & Swartz & x.xxe-X & x.xxe-X & x.xxe-x & x.xxe-X & $\mathrm{p}$ \\
\hline & PD & $1.07 \mathrm{e}-0$ & $5.78 \mathrm{e}-1$ & $2.08 \mathrm{e}-1$ & $2.07 \mathrm{e}-15$ & 0.42 \\
\hline \multicolumn{7}{|c|}{$L_{\infty}$} \\
\hline \multirow[t]{5}{*}{0.05} & $\mathrm{MOF}$ & $8.21 \mathrm{e}-1$ & $6.24 \mathrm{e}-1$ & $2.67 \mathrm{e}-1$ & $1.47 \mathrm{e}-14$ & ??? \\
\hline & LVIRA & $8.21 \mathrm{e}-1$ & $6.24 \mathrm{e}-1$ & $2.67 \mathrm{e}-1$ & $1.50 \mathrm{e}-14$ & ??? \\
\hline & Youngs & $8.22 \mathrm{e}-0$ & $6.25 \mathrm{e}-0$ & $2.67 \mathrm{e}-1$ & $1.46 \mathrm{e}-14$ & $? ? ?$ \\
\hline & Swartz & x.xxe-X & x.xxe-x & x.xxe-x & x.xxe-x & $\mathrm{p}$ \\
\hline & PD & $8.22 \mathrm{e}-1$ & $6.25 \mathrm{e}-1$ & $2.67 \mathrm{e}-1$ & $1.45 \mathrm{e}-14$ & ??? \\
\hline \multirow[t]{5}{*}{0.06} & MOF & $1.15 \mathrm{e}-0$ & $8.41 \mathrm{e}-0$ & $3.72 \mathrm{e}-1$ & $1.94 \mathrm{e}-14$ & ??? \\
\hline & LVIRA & $1.15 \mathrm{e}-0$ & $8.38 \mathrm{e}-0$ & $4.12 \mathrm{e}-1$ & $1.96 \mathrm{e}-14$ & ??? \\
\hline & Youngs & $1.15 \mathrm{e}-0$ & $8.39 \mathrm{e}-0$ & $4.04 \mathrm{e}-1$ & $1.93 \mathrm{e}-14$ & ??? \\
\hline & Swartz & x.xxe-x & x.xxe-x & x.xxe-x & x.xxe-x & $\mathrm{p}$ \\
\hline & PD & $1.15 \mathrm{e}-0$ & $8.39 \mathrm{e}-0$ & $4.05 \mathrm{e}-1$ & $1.96 \mathrm{e}-14$ & ??? \\
\hline
\end{tabular}

Table 6: $L_{1}, L_{2}$, and $L_{\infty}$ error for the $V$ velocity in the colliding bubbles problem 
is a sound technique, although others existing methods give comparable results. However, for multiple materials, the increase in accuracy and elimination of the artificial material ordering suggest significant benefits for utilizing MOF.

For strongly driven bouyant flows, the errors in the flow solution dominate and all the interface reconstruction techniques perform similarly.

\section{Acknowledgments}




\section{References}

[1] A. S. Almgren, J. B. Bell, P. Colella, L. H. Howell, And M. L. WelCOME, $A$ conservative adaptive projection method for the variable density incompressible Navier-Stokes equations, Journal of Computational Physics, 142 (1998), pp. 1-46.

[2] J. B. Bell AND D. L. MARCus, A second-order projection method for variable density flows, Journal of Computational Physics, 101 (2002), pp. 334-348.

[3] C. Calgaro, E. Creusé, and T. Goudon, An hybrid finite volume-finite element method for variable density incompressible flows, Journal of Computational Physics, 227 (2008), pp. 4671-4696.

[4] I.-L. Chern, J. Glimm, O. McBryan, B. Plohr, and S. Yaniv, Front tracking for gas dynamics, Journal of Computational Physics, 62 (1986), pp. 83-110.

[5] M. A. Christon, Dealing with pressure: FEM solution strategies for the pressure in time-dependent Navier-Stokes equations, International Journal for Numerical Methods in Fluids, 38 (2002), pp. 1177-1198.

[6] R. Danchin, Density-dependent incompressible viscous fluids in critical spaces, Proceedings of the Royal Society of Edinburgh, 133A (2003), pp. 1311-1334.

[7] V. Dyadechko and M. Shashkov, Moment-of-fluid interface reconstruction, Tech. Report LA-UR-05-7571, Los Alamos National Laboratory, Los Alamos, NM, 2005.

[8] — Multi-material interface reconstruction from the moment data, Tech. Report LAUR-06-5846, Los Alamos National Laboratory, Los Alamos, NM, 2006.

[9] J. H. Ferziger, Interfacial transfer in Tryggvason's method, International Journal for Numerical Methods in Fluids, 41 (2003), pp. 551-560.

[10] Y. Fraigneau, J.-L. Guermond, and L. Quartapelle, Approximation of variable density incompressible flows by means of finite element and finite volumes, Communications in Numerical Methods in Engineering, 17 (2001), pp. 893-902.

[11] P. M. GResho AND S. T. Chan, On the theory of semi-implicit projection methods for viscous incompressible flow and its implementation via a finite element method that also introduces a nearly consistent mass matrix. part 2: Implementation, International Journal for Numerical Methods in Fluids, 11 (1990), pp. 621-659.

[12] J.-L. Guermond and L. Quartapelle, A projection fem for variable density incompressible flows, Journal of Computational Physics, 165 (2000), pp. 167-188.

[13] T. J. R. Hughes And L. P. FrancA, A new finite element formulation for computational fluid dynamics: Vii. the Stokes problem with various well-posed boundary conditions: symmetric formulations that converge for all velocity/pressure spaces, Computer Methods in Applied Mechanics and Engineering, 65 (1987), pp. 85-96. 
[14] J. E. P. JR. AND E. G. PUCKett, Second-order accurate volume-of-fluid algorithms for tracking material interfaces, Journal of Computational Physics, 199 (2004), pp. 465502.

[15] M.-J. Li And M. ABdou, A variable-density projection method for interfacial flows, Numerical Heat Transfer, part B, 44 (2003), pp. 553-574.

[16] C. Liu and N. J. Walkington, Convergence of numerical approximations of the incompressible Navier-Stokes equations with variable density and viscosity, SIAM Journal of Numerical Analysis, 45, pp. 1287-1304.

[17] S. Osher AND R. P. FEDKIW, Level set methods: an overview and some recent results, Journal of Computational Physics, 169 (2001), pp. 463-502.

[18] E. G. Puckett, A. S. Almgren, J. B. Bell, D. L. Marcus, And W. J. Rider, A high-order projection method for tracking fluid interfaces in variable density incompressible flows, Journal of Computational Physics, 130 (1997), pp. 269-282.

[19] J.-H. Pyo And J. Shen, Gauge-Uzawa methods for incompressible flows with variable density, Journal of Computational Physics, 221 (2007), pp. 181-197.

[20] W. J. Rider And D. B. Kothe, Reconstructing volume tracking, Journal of Computational Physics, 141 (1998), pp. 112-152.

[21] M. Rudman, A volume-tracking method for incompressible multifluid flows with large density variations, International Journal for Numerical Methods in Fluids, 28 (1998), pp. 357-378.

[22] S. P. Schofield, R. V. Garimella, M. M. Francois, and R. Loubere, Material order independent interface reconstruction using power diagrams, International Journal for Numerical Methods in Fluids, 56 (2008), pp. 643-659.

[23] — A second-order accurate material order independent interface reconstruction technique for multi-material flow simulations, Journal of Computational Physics, 228 (2009), pp. $731-745$.

[24] B. K. SwARTz, The second-order sharpening of blurred smooth borders, Mathematics of Computation, 52 (1989), pp. 675-714.

[25] D. L. Youngs, Time dependent multi-material flow with large fluid distortion, in Numerical Methods for Fluid Dynamics, K. W. Morton and M. J. Baines, eds., Academic Press, 1982, pp. 273-285.

[26] —_, An interface tracking method for a 3d Eulerian hydrodynamics code, Tech. Report AWE/44/92/35, AWRE Design and Math Division, 1984. 